\title{
The NUT study: the effect of dietetic and oral nutritional interventions on survival and quality of life in patients with weight loss undergoing palliative chemotherapy for gastrointestinal (GI) or lung malignancy, a randomised controlled trial
}

\author{
C. Baldwin ${ }^{1}$, A. Spiro ${ }^{1}$, C. McGough ${ }^{2}$, A. R. Norman ${ }^{3}$, M. O'brien ${ }^{1}$, D. C. Cunningham ${ }^{1}$ \\ and H. J. N. Andreyev ${ }^{1}$ \\ Departments of ${ }^{1}$ Medicine, ${ }^{2}$ Dietetics and ${ }^{3}$ Computing, Royal Marsden Hospital, Fulham Road, London SW3 6JJ
}

Weight loss in patients with GI and lung cancers is common and is associated with shorter survival and poorer quality of life. Oral nutritional interventions to manage weight loss in a range of clinical conditions improve outcomes but data in cancer patients on the clinical benefits of nutritional intervention are inadequate. The aim of this study was to assess the effect of dietary advice and/or oral nutritional interventions on survival and quality of life of patients with weight loss and advanced GI and lung cancers.

Patients with weight loss, in whom radical therapy was not possible and who were instead to receive palliative chemotherapy for histologically proven GI or non-small-cell lung cancer or mesothelioma were randomly assigned to receive (1) no intervention (ad libitum food intake), (2) a nutritional supplement $(240 \mathrm{ml}, 2510 \mathrm{~kJ} / \mathrm{d})$ and a vitamin supplement, (3) dietary advice (an additional $2510 \mathrm{~kJ} / \mathrm{d})$ and (4) dietary advice plus a nutritional and vitamin supplement (additional $2510 \mathrm{~kJ} / \mathrm{d}$ from food plus $2510 \mathrm{~kJ}$ from a supplement) for $6 \mathrm{keeks}$ beginning before the start of chemotherapy. EORTC-C30 was used to assess quality of life at 6 weeks and 26 weeks. Patients were followed for 1 year.

Five centres recruited 358 patients between 2002 and 2006, 256 men and 102 women (age median 66 (range 24-88) years). A total of 254 had GI cancer (oesophago-gastric, $n$ 71; pancreas, $n$ 72; lower GI, $n$ 111) eighty-one non-small-cell lung cancer or mesothelioma (fourteen other). Ninety-six patients were randomised to group 1, eighty-six to group 2, ninety to group 3 and eighty-six to group 4 . The groups were balanced for baseline characteristics. Median follow up for all patients was 6.8 (range 0-50) months. The 1-year survival for all patients combined was $37.8 \%$ (95\% CI 32.2,43.4). There was no significant difference in survival between the intervention groups

\begin{tabular}{|c|c|c|c|c|c|}
\hline \multirow[b]{2}{*}{ Group } & \multicolumn{2}{|c|}{ 1-year overall survival } & \multicolumn{2}{|c|}{ HR adjusted } & \multirow[b]{2}{*}{$P$-value } \\
\hline & Percentage & $95 \% \mathrm{CI}$ & & $95 \% \mathrm{CI}$ & \\
\hline 1 No intervention & 35.2 & $24.7,45.5$ & 1 & & \\
\hline 2 Supplement only & 34.4 & $23.6,45.5$ & 1.13 & $0.77,1.67$ & 0.541 \\
\hline 3 Dietary advice only & 36.7 & $25.9,47.5$ & 1.21 & $0.81,1.80$ & 0.356 \\
\hline 4 Advice + Supplement & 45.6 & $33.6,56.8$ & 0.94 & $0.63,1.41$ & 0.761 \\
\hline
\end{tabular}

Site, extent of disease and performance status were significantly associated with differences in survival $(P<0.001)$. There were no significant differences in quality of life between any of the groups at baseline or at the 6- and 26-week time points.

Six-week intervention with targeted dietary advice and/or nutritional and vitamin supplements that sought to provide 600-1200 calories/d does not improve outcome or quality of life in patients with advanced cancer receiving palliative chemotherapy. 